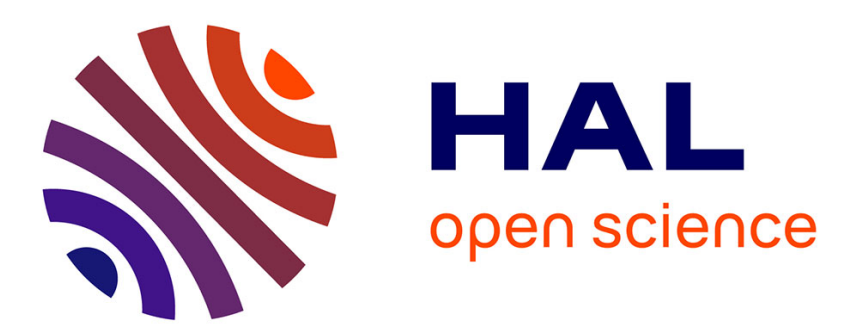

\title{
Kinetics of Adsorption of Linear Homopolymers onto Flat Surfaces: Rouse Dynamics
}

\author{
A. Semenov, J.-F. Joanny
}

\section{To cite this version:}

A. Semenov, J.-F. Joanny. Kinetics of Adsorption of Linear Homopolymers onto Flat Surfaces: Rouse Dynamics. Journal de Physique II, 1995, 5 (6), pp.859-876. 10.1051/jp2:1995169 . jpa-00248204

\section{HAL Id: jpa-00248204 https://hal.science/jpa-00248204}

Submitted on 1 Jan 1995

HAL is a multi-disciplinary open access archive for the deposit and dissemination of scientific research documents, whether they are published or not. The documents may come from teaching and research institutions in France or abroad, or from public or private research centers.
L'archive ouverte pluridisciplinaire HAL, est destinée au dépôt et à la diffusion de documents scientifiques de niveau recherche, publiés ou non, émanant des établissements d'enseignement et de recherche français ou étrangers, des laboratoires publics ou privés. 
Classification

Physics Abstracts

$61.25 \mathrm{H}-68.45-36.20$

\title{
Kinetics of Adsorption of Linear Homopolymers onto Flat Sur- faces: Rouse Dynamics
}

\author{
A. N. Semenov $\left({ }^{1,2}\right)$ and J.-F. Joanny $\left({ }^{1}\right)$ \\ ( ${ }^{1}$ Institut Charles Sadron (*), 6 rue Boussingault, 67083 Strasbourg Cedex, France \\ $\left({ }^{2}\right)$ Physics Department, Moscow State University, Moscow 117234, Russia
}

(Received 13 December 1994, received in final form and accepted 22 February 1995)

\begin{abstract}
The dynamic properties of unentangled adsorbed polymer layers are considered theoretically. The kinetics of penetration of a chain in an adsorbed layer is considered as a twostep process: entry corresponding to the attachment of the chain on the adsorbing surface and spreading on the surface. We discuss three types of experiments, the formation of an adsorbed layer, the desorption from an adsorbed layer and the exchange between an adsorbed layer and a bulk solution. The bottleneck for the adsorption of a new chain onto a solid wall (already covered by polymers) is the spreading stage corresponding to creation of $G$ contacts with the wall with a total binding energy of order of $k T$. The desorption of a preadsorbed layer in contact with pure solvent is extremely slow and only a very small fraction of the adsorbed chains is expected to desorb within the experimental time scale. The exchange between labelled adsorbed chains and unlabelled chains in solution is slower than the formation of a saturated layer, but much faster than the desorption process. The molecular weight dependencies of the characteristic times of the three processes are predicted using the Rouse-Zimm dynamics for the polymer chains.
\end{abstract}

\section{Introduction}

Adsorbed polymers play an important role in various areas of applied technologies such as lubrication adhesion or colloid stabilization. They have been the subject of many experimental and theoretical studies over the last years. A rather good description of the equilibrium properties of adsorbed polymer layers has been reached [1-3,17]. Polymers form on an adsorbing surface a fluffy layer with a thickness that can be as large as the radius of gyration of the polymer chains in solution. The polymer chains form loops on the surface with a very broad distribution of sizes [4]. The quantitative description of the polymer concentration and of the mechanical properties of polymer layers adsorbed from a good solvent are based on the self-similar structure proposed by De Gennes [1,2]. Many of the experimental results obtained from neutron scattering [5], neutron reflectivity, surface excess measurements or direct measurements of the forces between opposing layers are in good agreement with this scaling theory.

(*) UPR CNRS 022 
Recently [7], we have proposed a detailed scaling analysis of the statistics of loops and tails in a polymer layer adsorbed from a dilute good solvent where the tails are the end sections of the chains going from the surface to the bulk solution. We have shown that the layer has a double layer structure with an inner part where most of the monomers belong to loops and an outer part where most of the monomers belong to tails. This segregation between loops and tails was already observed in the numerical self-consistent mean-field theory of Scheutjens and Fleer $[3,25]$. We are not however aware of any direct experimental evidence of this structure (note however indirect experimental evidence [26]).

Most of the studies both theoretical and experimental devoted to polymer adsorption have considered it as a thermodynamic equilibrium process. The adsorption kinetics is however known to be a very slow process where the final equilibrium is reached only after very long times that may in many cases be longer than the accessible experimental time range. Polymer adsorption is often an irreversible process $[8,28,29]$. When an adsorbed layer is formed in equilibrium with a dilute solution and then exposed to a pure solvent, only a small fraction of the polymer chains desorbs. Most of the chains remain bound to the adsorbing surface. Thus it is of uppermost importance to build a detailed theory of the kinetics of polymer adsorption and desorption.

De Gennes $[10,11,15]$ has proposed a two-step process to describe the adsorption kinetics. In the first step, entry, a new polymer chain penetrating the adsorbed layer must overcome the potential barrier due to the excluded volume of the already adsorbed chains. This potential barrier is treated by analogy with a quantum tunneling problem. The spreading process corresponds to an increase of the number of monomers of the penetrating chain in contact with the adsorbing surface. The total number of monomers on the adsorbing surface increases only in the very early stages of the adsorption. In the later stages, the incoming chain replaces already adsorbed chains on the surface. The replaced chains unfold to form extra loops. Many of the observed qualitative features of polymer adsorption kinetics are in agreement with this theory: for example essentially no desorption is predicted but the exchange between labelled and unlabelled chains in the adsorbed layer occurs at a finite rate.

In this paper, we follow the lines of De Gennes's work to discuss the adsorption dynamics in more details. We assume that the adsorption is slow enough that at each step the adsorbed layer is in a quasi-equilibrium state. We do not consider the very early stages where a uniform polymer layer forms on the surface. The kinetics in these early stages is dominated by the bulk diffusion of the polymer towards the surface. In the later stages, we keep the same qualitative description as De Gennes with a two-step adsorption process, entry and spreading. The potential barrier opposing the penetration of new chains in the adsorbed layer is determined from our results on the statistics of loops and tails in the layer and turns out to be quite different from the one proposed by De Gennes. We explicitly take into account both the entry and the spreading mechanisms in the adsorption rate determination. We use these results to discuss three types of experiments: the kinetics of formation of an adsorbed layer, the kinetics of desorption and the exchange kinetics between labelled and unlabelled chains. In all this work, we ignore the role of entanglements on the dynamical properties of the polymer chains in the adsorbed layer and we use a Rouse-Zimm model to describe the dynamical properties of the polymers [20].

The paper is organized as follows. In the next section, we summarize De Gennes's scaling theory of equilibrium adsorbed polymer layers and our results on the structure of tails and loops. In Section 3, we discuss the penetration of a new chain in an adsorbed layer. We first discuss the potential barrier opposing the penetration and then the adsorption rate equation. Section 4 is devoted to the three basic experiments: adsorption, desorption and exchange. The last section presents our conclusions and discusses some possible issues. 


\section{Equilibrium Structure of an Adsorbed Layer}

In this section we present the self-similar construction proposed by De Gennes [1,2] to describe the structure of adsorbed polymer layers. We then summarize our results [7] on the statistics of tails and loops in the layer. Finally, we discuss the equilibrium between the adsorbed layer and a dilute good solvent.

2.1. Self-Similar Concentration Profile. - In the vicinity of an adsorbing surface, the concentration of a polymer solution decreases smoothly with the distance $z$ from the surface. Locally close to the wall the solution has the same mesh-like structure as a bulk semi-dilute solution with a local correlation length (mesh size) $\xi(z)$ that varies with the local polymer concentration $\phi(z)$ as $\xi(z) \sim \phi(z)^{-\nu /(3 \nu-1)}[12,13]$, where $\nu=0.588$ is the swelling exponent of a polymer chain in a good solvent. The distance from the wall being the only characteristic length scale in the problem, the self-similar construction proposed by De Gennes is obtained by imposing that the local correlation length be proportional to $z$. This gives the self-similar concentration profile as

$$
\phi(z) \sim z^{1 / \nu-3} \simeq z^{-4 / 3}
$$

(here and below the monomer size, a, is closer as unit length). In the limit of strong adsorption where each monomer in contact with the adsorbing surface gains an energy of the order of the thermal excitation $k T$, the short range cutoff for the concentration profile is of the order of the monomer size $a$. As discussed below, if the surface is saturated with polymer, the adsorbed layer cannot be thicker than the size of a free chain $R_{\mathrm{F}} \sim N^{\nu}$ in a bulk dilute solution. The self-similar concentration profile is thus observed up to a distance $R_{\mathrm{F}}$. For infinitely long chains, the surface coverage $\Gamma_{0}=\int \phi(z) \mathrm{d} z$ has a constant value of order $1 / a^{2}$.

Undersaturated or starved layers $[9,10]$ are obtained, either by stopping the adsorption process, or by forming an adsorbed layer in equilibrium with an extremely dilute solution. The surface excess is then smaller than the saturation value $\Gamma_{0}$. We define the undersaturation degree $x$ by

$$
\Gamma=\Gamma_{0}(1-x)
$$

The concentration profile has the same power law decay as for saturated layers but the thickness $l$ of an undersaturated layer is smaller than the chain radius $R_{F}[9,10]$. The explicit integration of the concentration profile leads to:

$$
l \simeq a x^{\frac{\nu}{1-2 \nu}} \simeq a x^{-3}
$$

where we suppose that $1>x>N^{1-2 \nu}$.

2.2. LOOPS AND TAILS. - De Gennes's scaling assumption for the concentration profile in an adsorbed polymer layer does not give any precise information on the single chain statistics in the layer. In general two types of chain sections are distinguished: loops are chain sections starting from the adsorbing surface and coming back to the adsorbing surface; tails are chain sections starting from the adsorbing surface and dangling towards the bulk of the solution. Here we summarize our recent results on the relative importance of loops and tails in an adsorbed layer.

An adsorbed layer has a double layer structure with an inner part where the concentration is essentially dominated by monomers belonging to loops and an outer part where the concentration profile is essentially dominated by monomers belonging to tails. The crossover between these two sublayers occurs at a distance $\tilde{z}$ from the adsorbing surface much smaller than the bulk radius of gyration $R_{\mathrm{F}}$

$$
\tilde{z} \sim N^{1 / 2}
$$


In the inner layer, where the loops dominate, the partition function $Z(g)$ of a loop containing $g$ monomers is directly related to the loop concentration (approximately equal to the total concentration) at a distance $z=g^{\nu} a$.

$$
z \phi_{1}(z) \sim g^{2} Z(g)
$$

The partition function of a loop can thus be written as [4]:

$$
Z(g) \sim g^{-1-2 \nu}
$$

The partition function of a tail can be obtained by considering a loop of $2 g$ monomers as two half tails each containing $g$ monomers and meeting at their end point. The following relation is then obtained between the partition functions of loops and tails

$$
Z(g) \sim g^{1-\gamma-\nu} Z_{\mathrm{t}}^{2}(g)
$$

The exponent $\gamma=1.162$ is the susceptibility exponent [16]. The partition function of a tail is therefore

$$
Z_{\mathrm{t}}(g) \sim g^{(\gamma-\nu) / 2-1}
$$

From the partition function of a tail one obtains the concentration of monomers belonging to tails

$$
\phi_{\mathbf{t}}(z) \sim N^{-1} z^{1 / \nu-1}
$$

The tail concentration is small in the very vicinity of the adsorbing surface where many loops are formed; it increases with the distance from the wall and becomes of the order of the total concentration at a distance of order $\tilde{z}$. At larger distances, the concentration of monomers belonging to tails is larger than that of monomers belonging to loops.

In the outer layer where tails dominate $(z>\tilde{z})$, the partition function of tails is directly related to the total concentration by

$$
z \phi(z) \sim \operatorname{const} g^{2} Z_{\mathrm{t}}(g)
$$

where const is $N$-dependent. Imposing a good crossover at $z=\tilde{z}$, we obtain

$$
Z_{\mathrm{t}}(g) \sim N^{1+\frac{\gamma-\nu}{4 \nu}} g^{-1-2 \nu}
$$

One can then calculate the partition function of a loop and the concentration of monomers belonging to loops, $\phi_{1}(z) \sim N^{\frac{3}{2}+\frac{\gamma}{2 \nu}} z^{-6+\frac{1-\gamma}{\nu}}$. One easily checks that the loop concentration is much smaller than the total concentration in this outer layer and that the concentration profile is indeed dominated by tails.

These predictions can be easily generalized for $d$ dimensions. The final results are

$$
\phi_{1}(z) \sim\left\{\begin{array}{cl}
z^{1 / \nu-d} & , \quad z<\tilde{z}=N^{\frac{1}{d-1}} \\
N^{\frac{d+\gamma / \nu}{d-1}} z^{-2 d+\frac{1-\gamma}{\nu}}, & \tilde{z}<z<l=G^{\nu}
\end{array}\right.
$$

for loops, and

$$
\phi_{\mathrm{t}}(z) \sim\left\{\begin{array}{cc}
N^{-1} z^{1 / \nu-1} & , \quad z<\tilde{z} \\
z^{1 / \nu-d} & , \quad \tilde{z}<z<l
\end{array}\right.
$$

for tails where $G$ is the maximum size of the tails or loops. Note that $d=4, \gamma=1, \nu=1 / 2$ correspond to the mean-field predictions, whereas $d=3, \gamma=1, \nu=1 / 2$ correspond to an adsorbed layer in a theta solvent.

For a starved layer, if the layer thickness $l$ is smaller than the crossover thickness $\tilde{z}$, the outer region dominated by the tails does not exist. 
2.3. Adsorbed Polymer Layer in Equilibrium With a Dilute Solution. - The overall structure of an adsorbed polymer layer is the result of a thermal equilibrium between the adsorbed chains and the free chains in the bulk solution. We now consider this equilibrium when the polymer in the bulk is dilute and in a good solvent.

In order to determine the chemical potential of the polymer in the adsorbed layer, we consider the adsorbed layer as a two-dimensional melt of blobs. For a two-dimensional melt with a density of chains $\Gamma / N$, the free energy per unit area can be written as

$$
F / k T=f(\Gamma)+\frac{\Gamma}{N} \ln \frac{\Gamma}{e N}-\frac{\Gamma}{N} \ln \left(N^{\gamma_{2 D}-1}\right)
$$

The first term depends only on the monomer two-dimensional density $\Gamma$ and not on the molecular weight $N$, it describes the interactions between monomers. The second term represents the translational entropy of the chains and the last term is the configurational entropy of each chain in a two-dimensional melt. The susceptiblity exponent $\gamma_{2 \mathrm{D}}$ is exactly known in two dimensions from conformal invariance arguments [14]

$$
\gamma_{2 \mathrm{D}}-1=3 / 16
$$

An adsorbed polymer layer is a melt of blobs of varying size formed by the loops and tails. In each adsorbed chain, a finite fraction of the monomers are in direct contact with the adsorbing surface and the number of loops per chain is of order $N$. The direct contribution of the loops to the free energy is dominated by short loops and is thus independent of molecular weight. The loop contribution to the free energy can therefore be incorporated in the interaction term $f(\Gamma)$. The tail contribution to the free energy is directly calculated from the tail partition function

$$
F_{\mathrm{t}}=-2 k T \ln \left(Z_{\mathrm{S}}\right)
$$

The factor 2 accounts for the existence of two tails in each chain and the relevant partition function is obtained by integrating the tail partition function over the tail length.

$$
Z_{\mathrm{S}}=\int Z_{\mathrm{t}}\left(g^{\prime}\right) \mathrm{d} g^{\prime} \sim N^{\frac{\gamma-\nu}{4 \nu}}
$$

Here we have assumed that the undersaturation is small enough so that size of the largest tails $G \sim l^{1 / \nu}$ is larger than the crossover size $\tilde{g}=\tilde{z}^{1 / \nu} \sim N^{1 /(2 \nu)}$. The partition function $Z_{\mathrm{S}}$ is dominated by tails of size $\tilde{g}$.

The existence of tails and loops could also renormalize the exponent $\gamma_{2 \mathrm{D}}$; this has not been studied to our knowledge and we shall in the following use here the melt value. The free energy per unit area of the adsorbed layer reads then

$$
F_{\text {ads }}=f(\Gamma)+\frac{\Gamma}{N} \ln \frac{\Gamma}{e N}-\frac{\Gamma}{N} A \ln N
$$

where $A=\gamma_{2 \mathrm{D}}-1+\frac{\gamma-\nu}{2 \nu}$, and where we have chosen $k T$ as the energy unit here and below. The chemical potential per monomer is

$$
\mu_{\mathrm{ads}} \equiv \frac{\partial F_{\mathrm{ads}}}{\partial \Gamma}=-\Delta \mu(x)+\frac{1}{N} \ln \frac{\Gamma}{N}-\frac{A}{N} \ln N
$$

$\Delta \mu(x) \equiv-\frac{\partial f}{\partial \Gamma}$ is the effective attraction energy of one monomer on the adsorbing wall. If the layer is starved, this chemical potential term is dominant since all the other terms decrease as $1 / N$. The formation of a large tail or loop of $\mathrm{g}$ monomers costs an energy $g \Delta \mu$. The maximum 
tail or loop size that fixes the thickness of the layer, obtained when this energy is of order $k T$ is

$$
G=1 / \Delta \mu
$$

The chemical potential of a monomer in a dilute solution of concentration $c$ smaller than the overlap concentration $c^{*} \sim N^{1-3 \nu}$ is

$$
\mu_{\text {sol }}=\frac{1}{N} \ln \frac{c}{N}-\frac{1}{N} \ln N^{\gamma-1}
$$

where the first term is the translational entropy and the last term is the configurational entropy of the chains $[12,13]$.

The balance of the chemical potential in the bulk and on the surface leads to

$$
N / G \equiv N \Delta \mu=A^{\prime} \ln N-\ln \frac{c}{c^{*}}
$$

where $A^{\prime}=\gamma-\gamma_{2 \mathrm{D}}+3 \nu-(1+\gamma / \nu) / 2 \simeq 0.25$. In the vicinity of the overlap concentration, the maximum tail size is $G=\frac{1}{\Delta \mu} \simeq \frac{4 N}{\ln N}$ and the layer thickness $l \sim G^{\nu} a$ is indeed of the order of the radius of gyration of isolated polymers in a good solvent.

\section{Penetration of a Chain in an Adsorbed Polymer Layer}

We now discuss the penetration of a new chain in the adsorbed layer. We ignore here the early stages of the adsorption where a uniform continuous layer is formed on the surface. The adsorption kinetics is in this stage limited by the bulk diffusion of polymer chains towards the surface and shows no specific polymeric effect. In the following stages, there is a homogeneous polymer layer on the adsorbing surface where already adsorbed chains form loops and tails. We assume throughout the paper that this layer has the equilibrium structure of a starved layer as described in the previous section. It is thus characterized by an undersaturation degree $x$ or equivalently by the size of the largest tails or loops $G \sim x^{\frac{1}{1-2 \nu}}$. This equilibrium assumption is valid if the incoming flux of adsorbing chains is small enough that at each step the layer can relax to its equilibrium structure. Below we show (see the end of Section 4.1) that this local equilibrium assumption is always valid for a Rouse adsorption from a dilute solution.

We first consider the potential barrier seen by a penetrating polymer chain: the penetration is done in two steps, entry where the penetrating chain has no monomer in direct contact with the surface and sees the excluded volume due to the already adsorbed chains and spreading where the monomers of the penetrating chain come into contact with the adsorbing surface. During the spreading step, the penetrating chain must expell from the surface the already adsorbed chains that form larger loops or tails. A chain is irreversibly adsorbed if it has gained an energy larger than $k T$ upon adsorption. This is the case if the number of adsorbed monomers $g$ in the chain (i.e., the monomers involved in loops) is such that $g \Delta \mu \gg 1$. A chain is thus considered as irreversibly adsorbed when the number of adsorbed monomers is larger than the cutoff number $G$. At later times the chain relaxes towards its equilibrium structure of tails and loops.

We then discuss the dynamical resistance of the potential barrier that fixes the flux of incoming chains. We use here Kramers rate [24] theory and the Zimm-Rouse description of the dynamics of polymer chains [20].

\subsection{Potential Barrier}

3.1.1. Tails and Hairpins Partition Functions. - In order to study the entry of a new chain in the adsorbed layer, we first determine the partition function of a chain touching the surface by 

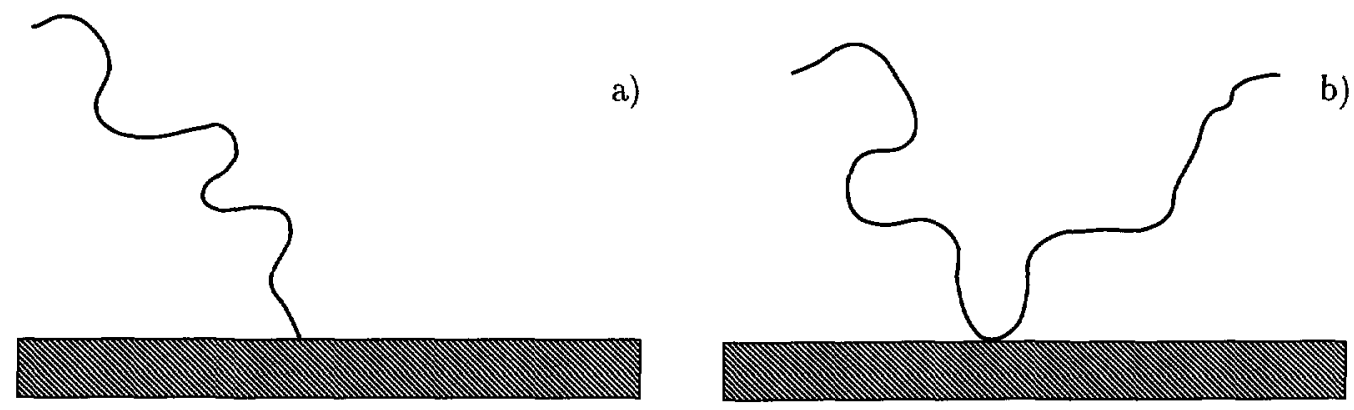

Fig. 1. - a) end entry; b) hairpin entry.

a single monomer. We distinguish the case where this monomer is one of the end monomers of the chain that we call end entry (Fig. 1a) and the case where this monomer is a monomer in the middle of the chain that we call hairpin entry (Fig. 1b).

If the number $N$ of monomers in the chain is equal to the maximum tail size $G$, the partition function of the chain attached by one end $Z^{e}$ is the partition function of a tail of $G$ monomers $Z_{\mathrm{t}}(G)$ given by equations (2.8) or (2.10) when $G$ is smaller or larger than the crossover size $\tilde{g}$, respectively. If the entering chain is much larger than $G$, it forms a very long tail outside the adsorbed layer and it behaves as a free chain attached to a hard repulsive wall. The partition function of such a tail is $Z=N^{\gamma_{1}-1}$ where $\gamma_{1}$ is a surface exponent introduced for polymers by Duplantier [14]. There is no precise numerical estimate of this exponent in three dimensions: the $\epsilon$ expansion in dimension $d=4-\epsilon$ is known to first order $\gamma_{1}-1=-1 / 2+\epsilon / 8$; in two dimensions the exact value is known from conformal theory $\gamma_{1}-1=-3 / 64$. We have extrapolated between these two values by assuming a quadratic variation of $\gamma_{1}$ with the space dimension. We obtain the approximate value

$$
\gamma_{1} \simeq 0.676
$$

These two limiting behaviors impose the scaling form of the partition function of a chain attached by one end in the adsorbed layer

$$
Z^{\mathrm{e}}=(N / G)^{\gamma_{1}-1} Z_{\mathrm{t}}(G)
$$

The penetration of a chain in the adsorbed layer with a front monomer close to the end point is very similar to the end-point penetration. Thermal fluctuations then favor the existence of a short tail (shorter than $G$ ) during penetration. The partition function of a chain forming such a small tail is obtained by multiplying the partition function of a chain touching the surface by its end point by the partition function of a tail with any length $g$ (where $0<g<G$ ), $Z_{\mathrm{S}}(G)=\int_{0}^{G} Z_{\mathrm{t}}(g) \mathrm{d} g$ (compare with Eq. (2.15)):

$$
Z^{e t}=(N / G)^{\gamma_{1}-1} Z_{\mathrm{t}}(G) Z_{\mathrm{S}}(G)
$$

As already noted if $G>\tilde{g}$, the average tail size is given by the crossover value $\tilde{g}$. We thus expect that when a chain touches the surface close to its end point, it forms on average a small tail of size $\tilde{g}$.

The other possibility is that the chain touches the surface by one of its middle points to make a hairpin. It then has two long tails going outside the adsorbed layer. If the number of monomers $N$ is of order $G$, the chain is equivalent to two tails and its partition function is 
$G Z_{\mathrm{t}}^{2}(G)$ where the extra factor $G$ accounts for the fact that any of the monomers can be in contact with the surface. If the chain is very long, it behaves as a chain attached to a repulsive hard wall by any of its points. The partition function of a chain touching a hard wall by any middle point [14] does not involve any surface exponent, it varies as $Z \sim N^{\gamma-1}$. If only the closest monomer touches a repulsive surface, the number of configurations of the chain is not significantly reduced and, the partition function is the same as that of a free chain. Looking for a scaling law, we find the partition function of a hairpin touching the adsorbing wall by one monomer as

$$
Z^{\mathrm{hp}}=(N / G)^{\gamma-1} G Z_{\mathrm{t}}^{2}(G)
$$

3.1.2. End and Hairpin Entry. - We now determine the potential barrier seen by a polymer chain during the entry process. As in the previous section, we consider two different entry mechanisms: end entry and hairpin entry. In all this section, we label the position of the penetrating chain by the position $z$ of the closest monomer to the adsorbing surface. It turns out to be more convenient below to use the coordinate $g=(z / a)^{\nu}$. The potential barrier $U(z)$ seen by the entering chains is such that $\exp (-U(z))=Z(z) / Z_{0}$ where $Z(z)$ is the partition function of a chain with its closest monomer at a distance $z$ from the surface and $Z_{0} \sim N^{\gamma-1}$ is the partition function of an isolated chain in a good solvent. The partition function $Z(z)$ is inferred here from scaling arguments.

We first consider the end entry process in the case where the chain end is the monomer closest to the surface and when the undersaturation is large enough, $G<\tilde{g}$. When the end monomer is on the adsorbing surface $z=0$ the partition function is given by equation (3.2), $Z(z)=Z^{\mathrm{e}}$. When the end point is on the surface of the adsorbing layer, $g=G$, the entering chain can be considered as a chain of blobs of size $G$ attached to an infinitely repulsive surface by its end point and $Z(z=l)=G^{\gamma-1}(N / G)^{\gamma_{1}-1}$. Assuming a power law behavior in $g$ between these two limits we find for $g<G$

$$
Z(z)=g^{\gamma-1} \frac{Z^{\mathrm{e}}}{Z_{\mathrm{t}}(g)}
$$

where $Z_{\mathrm{t}}(g)$ is the partition function of a tail of $g$ monomers inside the adsorbed layer given by equation (2.8). When the distance of the end point from the wall is equal to the radius $R_{\mathrm{F}}$ of a free chain, the wall has no influence on the chain configurations and the partition function is that of a free chain $Z_{0}$. Looking for a scaling law, we find the partition function $Z(z)$ for $G<g<N$

$$
Z(z)=N^{\gamma_{1}-1} g^{\gamma-\gamma_{1}}
$$

The potential barrier for end entry when $G<\tilde{g}$ can thus be written as

$$
\exp (-U(z))=(g / N)^{\gamma-1} \frac{Z^{\mathrm{e}}}{Z^{\mathrm{e}}(g)}
$$

where we have defined $Z^{\mathrm{e}}(g)$ as the tail partition function $Z_{\mathrm{t}}(g)$ if $g<G$ and as the partition function of a chain of $g$ monomers with its end point attached to the adsorbing surface (given by Eq. (3.2) where $N$ is replaced by $g$ ) when $G<g<N$.

When the undersaturation is lower and $G>\tilde{g}$, the potential barrier can be determined using the same procedure. One must here impose that when $g<\tilde{g}$ the partition function has the same $g$ dependence as when $G<\tilde{g}$. Imposing the same boundary conditions as above when $g=0, g=G$ and $g=N$, we find that the potential barrier $\exp (-U(z))$ is still given by equation (3.7) where the tail partition function is given by equation (2.8) if $g<\tilde{g}$, and by equation (2.10) if $G>g>\tilde{g}$. 
As seen in the previous section, it is more favorable for the entering chain to attach to the surface not precisely by its end point but to make a small tail with an average number of monomers of order $\tilde{g}$. Using the same kind of scaling argument as above, we find the potential barrier for end entry taking into account this small tail. We assume below that $g<G$ since for $g>G$ the adsorbed layer is unimportant. If $G<\tilde{g}$

$$
\exp (-U(z))=(g / N)^{\gamma-1} \frac{Z^{\mathrm{e}} Z_{\mathrm{S}}(G)}{Z^{\mathrm{e}}(g) Z_{\mathrm{S}}(g)}
$$

where $Z_{\mathrm{S}}(g)$ is the integrated partition function of the tails given by equation (2.15), with an upper integration bound $g$. For lower undersaturations, $G>\tilde{g}$, when $g<\tilde{g}$ the potential barrier is given by

$$
\exp (-U(z))=(g / N)^{\gamma-1} \frac{Z^{\mathrm{e}} Z_{\mathrm{S}}(\tilde{g})}{Z^{\mathrm{e}}(g) Z_{\mathrm{S}}(g)}
$$

When $\tilde{g}<g<G$, the small tail does not play any role and the potential barrier is given by equation (3.7).

The potential barrier is constructed in a similar manner in the case of hairpin entry. The scaling analysis leads to

$$
\exp (-U(z))=(g / N)^{\gamma-1} \frac{Z^{\mathrm{hp}}}{Z^{\mathrm{hp}}(g)}
$$

where we have defined the partition function of a hairpin of $g$ monomers $Z^{\mathrm{hp}}(g)$ as $Z^{\mathrm{hp}}(g)=$ $g Z_{\mathrm{t}}^{2}(g)$ if $g<G$ and by equation (3.4) where $N$ is replaced by $g$ if $g>G$.

Both for end entry and hairpin entry we thus find a potential such that $\exp (-U(z))$ decreases as a power law of the distance between the closest monomer and the surface. The repulsion due to the excluded volume interaction decays therefore logarithmically from the surface. The prefactor of the logarithmic decrease is a complicated combination of the exponents $\gamma, \nu$ and $\gamma_{1}$. The numerical value of the prefactor turns out to be of some importance in the determination of the adsorption rate. The maximum of the potential seen by the penetrating chain occurs when the first monomer touches the adsorbing surface. The height of the maximum (given by Eqs. (3.9) and (3.10)) is larger for hairpin entry for weakly starved layers $G>g^{*}$ and larger for end entry for more starved layers. The crossover occurs at

$$
g^{*}=N^{a} \quad \text { where } \quad a=\left(1+\gamma-\gamma_{1}\right) /\left(\gamma+2 \nu-\gamma_{1}\right)=0.89
$$

Note that $g^{*}$ is larger than the crossover size $\tilde{g}$.

3.1.3. Spreading. - When the first monomer of the chain touches the surface, the chain spreads onto the surface to form a train and more and more monomers come in contact with the surface and become involved in loops. We characterize the configuration of the chain by the number $g$ of monomers forming a train on the surface which is proportional to the number of monomers in direct contact with the adsorbing surface. The partition function of the penetrating chain can then be written as $Z(g)=Z(g=1) Z^{\mathrm{sp}}(g)$ where $Z(g=1)$ is the partition function of a chain with one monomer in contact with the surface that we just determined and $Z^{\mathrm{sp}}(g)$ is the partition function of a chain of $g$ monomers adsorbed on the surface with no tail. Following the lines of Section 3.3, we write this partition function as

$$
Z^{\text {sp }}(g)=\mathrm{e}^{g \Delta \mu} g^{\gamma_{2 \mathrm{D}}-1}
$$

The potential seen by the penetrating chain is then

$$
\exp (-U(g))=\mathrm{e}^{g \Delta \mu} g^{\gamma_{2 \mathrm{D}}-1} N^{1-\gamma} Z(g=1)
$$


The partition function $Z(g=1)$ is given by $Z^{\text {et }}$, equation (3.3) when the penetration occurs by end entry and by $Z^{\mathrm{hp}}$, equation (3.4), when the penetration occurs by hairpin entry. If only a few monomers are adsorbed on the surface, $g<G=1 / \Delta \mu$ the potential decreases logarithmically as $g$ increases. When many monomers are adsorbed $g>>$ the chain is irreversibly adsorbed and the potential decreases linearly with $g$.

3.2. AdsORPtion RATE. - We now discuss the dynamics of penetration of new chains into the adsorbed layer. The flux $J$ of monomers belonging to newly adsorbed chains is limited by the diffusion through the potential barrier that we just described. As the barrier involved is larger than $k T$, we use Kramers rate theory [24] that characterizes the potential barrier by a resistance $K$ which relates the average incoming flux to the external monomer concentration $c, c=K J$. If we define a reaction coordinate $X$, the resistance of the barrier is given by

$$
K=\int \frac{\exp (U)}{D^{*}} \mathrm{~d} X
$$

where $U$ is the potential and $D^{*}$ is the effective diffusion constant.

As above, we split the potential barrier into two parts corresponding to the entry and the spreading processes and write the resistance as a sum of two terms, $K=K_{\mathrm{e}}+K_{\mathrm{sp}}$. For the entry process, we use the position $z$ of the monomer closest to the surface as the reaction coordinate; for the spreading process, we use the number $g$ of monomers of the penetrating chain in direct contact with the wall.

3.2.1. Rouse Dynamics. - The dynamics of polymer chains in an adsorbed layer strongly depends on the presence of entanglements. Although, for static purposes, the layer essentially behaves as a two-dimensional system (where no entanglement is possible), it is quite possible that the polymer chains are entangled (if the molecular weight is high enough) since the thickness of the layer is larger than the monomer size, $a$. Moreover, we expect the number of monomers per entanglement, $N_{\mathrm{e}}$, in an adsorbed layer to be of the same order as in the bulk. For actual experiments, the molecular weights are often in the entangled regime, $N>N_{\mathrm{e}}[2,17]$. This regime was considered by De Gennes $[10,11]$ within the framework of the reptation theory. However from the theoretical point of view, the entangled regime is more complex in these twodimensional systems [18] and the classical reptation picture [19,20] cannot be used as such. The reptation dynamics is considerably slowed down by a coupling with excluded-volume interactions which have a very strong effect in $2 \mathrm{D}$ systems. Recent results indicate that the disentanglement time, $\tau_{\mathrm{d} \text { ss }}$, is very long [21] and increases exponentially with the molecular weight $N>N_{\mathrm{e}}$ :

$$
\tau_{\text {dis }} \sim \exp \left(\text { const } N / N_{\mathrm{e}}\right)
$$

We therefore expect an extremely slow relaxation of a nearly saturated layer to its equilibrium state when $N \gg N_{\mathrm{e}}$. The relaxation time might in some cases be much longer than the time of the experiment. This stresses the importance of a detailed study of the structure and dynamics of non-equilibrium layers. We leave this problem for future efforts. In the present paper, as a first step, we simply assume that the adsorbed polymer layer is not entangled, $N<N_{\mathrm{e}}$.

We thus use the Rouse-Zimm dynamics to describe the motion of the polymer chains in the layer. Locally the adsorbed layer has a structure similar to that of a semidilute polymer solution and the hydrodynamic screening length is proportional to the local correlation length [20], i.e., to the distance $z$ from the wall. The friction constant of a loop or a tail containing $g=(z / a)^{1 / \nu}$ monomers is $\zeta=6 \pi \eta z$ where $\eta$ is the solvent viscosity. In the vicinity of the adsorbing surface, there are mostly small loops of size $a$ and the screening length is of the order of the monomer size; the friction constant per monomer is then of order $\zeta_{0}=6 \pi \eta a$. 
3.2.2. Entry. - The resistance of the potential barrier due to the entry process is given by

$$
K_{\mathrm{e}}=\int_{0}^{R_{\mathrm{F}}} \mathrm{d} z \mathrm{e}^{U(z)} / D(z)
$$

The relevant diffusion constant $D(z)$ is inversely proportional to the friction constant related to a movement of the front monomer from a distance $z$ to the surface, $D=k T / \zeta(z)$. This motion does not require a motion of the chain as a whole but only that of a piece of chain (blob) of size $z$. The friction constant is thus $\zeta(z)=6 \pi \eta z$, therefore the diffusion constant $D=k T /(6 \pi \eta z)$. In the limit of small undersaturation, $G>\tilde{g}$ the integral giving the resistance of the potential barrier has two contributions one coming from the large distances $z=R_{\mathrm{F}}$ and one coming from distances of the order of the crossover distance $\tilde{z}$.

For end entry we find:

$$
K_{e}^{\mathrm{e}}=6 \pi \eta\left[N^{2 \nu}+N^{\gamma-\gamma_{1}-\frac{\gamma}{2 \nu}} G^{\gamma_{1}+2 \nu}\right]
$$

The existence of a small tail does not influence this result. The first contribution is that of a transparent potential barrier $U \simeq 0$. The exluded volume interaction limits the penetration of new chains inside the adsorbed layer only in the case where the second term dominates, i.e., when the size of the biggest tails $G$ is large enough $G>N^{\frac{2 \nu+\gamma_{1}+\frac{\gamma}{2 \nu}-\gamma}{\gamma_{1}+2 \nu}} \sim N^{0.905}$. For hairpin entry, we find

$$
K_{\mathrm{e}}^{\mathrm{hp}}=6 \pi \eta\left[N^{2 \nu}+N^{-1-\frac{\gamma}{2 \nu}} G^{\gamma+4 \nu}\right]
$$

In the case of hairpin entry, also, the potential barrier is not transparent only at large values of the maximum loop size $G>N^{\frac{2 \nu+1+\frac{\gamma}{2 \nu}}{\gamma+4 \nu}} \sim N^{0900}$.

If the undersaturation is large $G<\tilde{g}$, the potential barrier is always transparent; the potential barrier is too weak to reduce the flux of incoming chains.

3.2.3. Spreading. - The contribution of the spreading process to the resistance of the potential barrier is

$$
K_{\mathrm{sp}}=\int \mathrm{d} g \mathrm{e}^{U(g)} / D(g)
$$

where $U(g)$ is determined by equation (3.13). The effective diffusion constant is now determined by estimating the dissipation associated to the spreading process. The dissipation has two origins: a direct friction on the incoming monomers (directly given by the Rouse dynamics) and the dissipation associated with the expulsion of the already adsorbed chains from the area when the penetrating chain spreads.

Polymer chains in a two-dimensional melt obey nearly-Gaussian statistics (apart from logarithmic corrections): in particular, the end-to-end distance of a chain is roughly proportional to the square root of the number of monomers [23]. Thus the area occupied on the adsorbing surface by a chain which has spread over $g$ monomers is $A \sim g$. The 2D concentration, $g / A$, in the occupied region is thus of order unity: the penetrating chain pushes a considerable fraction of the monomers of the already adsorbed chains out of this region. The spreading implies then an outward $2 \mathrm{D}$ flow of the monomers. Assuming that the $2 \mathrm{D}$ concentration in the adsorbed layer is nearly fixed, i.e., that the layer is $2 \mathrm{D}$-incompressible, we find that the flux $\jmath=2 \pi \Gamma v r$ of monomers through a circle of radius $r$ is nearly constant (here $v$ is the typical velocity of the flow at the distance $r$ from the center of the region). Therefore $v \sim \jmath /(\Gamma r)$, where $\jmath=\mathrm{d} g / \mathrm{d} t$ is the flux of "new" monomers into the layer. The total dissipation due to this flow is

$$
\mathcal{D}=\zeta_{0} \int_{R}^{R_{\max }} \Gamma v(r)^{2} 2 \pi r \mathrm{~d} r \sim \frac{\zeta_{0} \jmath^{2}}{\Gamma} \ln \left(R_{\max } / R\right)
$$



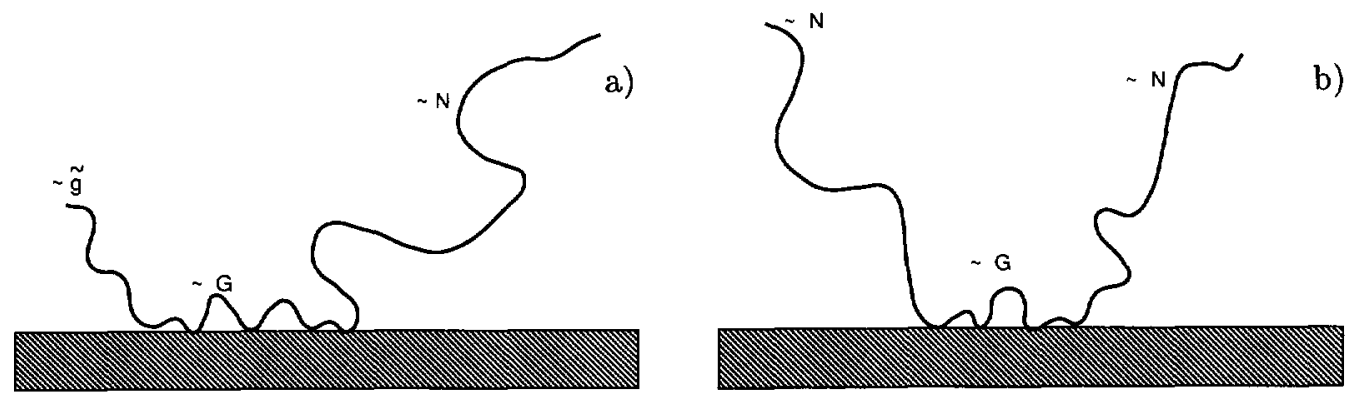

Fig. 2. - A typical chain conformation after spreading of $\sim G$ monomers ("critical" spreading): a) for end entry; b) for hairpin entry.

where $\zeta_{0}=6 \pi \eta a$ is the bare Rouse friction and where $R_{\max }$ is the long-range cut-off for the flow due to the small compressibility of the layer. The logarithmic factor in equation (3.20) always has a value between $\sim 1$ and $\sim \ln N$. In the following scaling estimates, this factor is omitted. Thus we simplify equation (3.20) as

$$
\mathcal{D} \sim \frac{\zeta_{0}}{\Gamma}\left(\frac{\mathrm{d} g}{\mathrm{~d} t}\right)^{2}
$$

The effective friction due to the expulsion of the other chains is thus of the same order as the direct Rouse friction and the spreading process can be roughly considered as an ordinary (Fickian) diffusion along the "coordinate" $g$, with the effective diffusion constant $D_{0}=k T \Gamma / \zeta_{0}$.

The contribution of the spreading process to the potential barrier resistance is dominated by a number of spread monomers of the order of the size of the largest tails $G$. Thus we find using equations (3.19) and (3.13) and assuming that $\Gamma \sim 1$ :

$$
K_{\mathrm{sp}}=6 \pi \eta\left[N^{\gamma-1} G^{2-\gamma_{2} \mathrm{D}}\right] / Z(g=1)
$$

The typical conformations with $\sim G$ spreaded monomers after end entry and after hairpin entry are shown in Figure $2 a$ and $2 b$. When $G>\tilde{g}$, if the penetration occurs by end entry, the resistance is

$$
K_{\mathrm{sp}}^{\mathrm{e}}=6 \pi \eta N^{\gamma-1 / 2-\gamma_{1}-\frac{\gamma}{2 \nu}} G^{2-\gamma_{2 D}+\gamma_{1}+2 \nu} \sim N^{-1.00} G^{2.66}
$$

If the penetration occurs by hairpin entry

$$
K_{\mathrm{sp}}^{\mathrm{hp}}=6 \pi \eta N^{-3 / 2-\frac{\gamma}{2 \nu}} G^{2-\gamma_{2 D}+\gamma+4 \nu} \sim N^{-2.48} G^{4.32}
$$

The total resistance of the potential barrier can now be determined from equations (3.17), (3.18), (3.22) and (3.23). The mechanism (end or hairpin penetration) that leads to the lowest resistance is the dominant mechanism. One can easily see that the total resistance is always dominated by the spreading process. Hairpin entry is favored if $G<g^{*}$, where $g^{*} \simeq N^{0.89}$ is given by equation (3.11), and end entry is favored if $G>g^{*}$.

In the region $G<\tilde{g}$ the entry barrier is transparent: $K_{\mathrm{e}}=K_{\mathrm{e}}^{(0)} \sim 6 \pi \eta N^{2 \nu}$. The hairpin spreading resistance for $G<\tilde{g}$ is determined by equations (3.19),(3.13) and (3.4):

$$
K_{\mathrm{sp}}^{\mathrm{hp}} \sim 6 \pi \eta G^{2-\gamma_{2 \mathrm{D}}+\nu}
$$

Thus the barrier for penetration is completely transparent if $K_{\mathrm{sp}}^{\mathrm{hp}}<K_{\mathrm{e}}^{(0)}$, i.e., if $G<g_{1}=$ $N^{\frac{2 \nu}{\nu+2-\gamma_{2 d}}} \simeq N^{0.84}$. Note that $g_{1}$ is very close to $\tilde{g}=N^{\frac{1}{2 \nu}} \simeq N^{085}$. For $G>g_{1}$ the resistance is determined by the spreading process. 
In the following, we focus on weakly undersaturated layers where $G$ is large and the potential barrier resistance is dominated by the spreading process and end penetration is favored, $K=$ $K_{\mathrm{sp}}^{\mathrm{e}}$ (Eq. (3.22)).

\section{Adsorption, Desorption and Exchange}

We now discuss the three basic experiments, formation of an adsorbed layer, desorption and exchange between adsorbate and solution, for a weakly starved layer where $G>g^{*}$ (defined by Eq. (3.11)) exposed to a dilute solution of the same polymer. The variation with time of the surface coverage, $\Gamma$, is due to the adsorption of new chains from the solution that create an inward flux $J_{+}$and to the desorption from the layer to the solution that creates an outward flux $J_{-}$:

$$
\frac{\mathrm{d} \Gamma}{\mathrm{d} t}=J_{+}-J_{-}
$$

The inward flux $J_{+}$is monitored by the resistance of the potential barrier given by equation (3.22):

$$
J_{+}=c / K_{\mathrm{sp}}^{\mathrm{e}} \simeq \rho N^{-3 \nu-\gamma+3 / 2+\gamma_{1}+\frac{\gamma}{2 \nu}} G^{-2+\gamma_{2 \mathrm{D}}-\gamma_{1}-2 \nu} \simeq \rho N^{0.24} G^{-266}
$$

where $\rho=c / c^{*}$ is the reduced concentration of the solution and where we have chosen $\tau_{0}=$ $\zeta_{0} a^{2} /(k T)$ as the unit time.

In order to calculate the outward flux $J_{-}$we note that at equilibrium the inward and outward fluxes are equal $J_{+}=J_{-}$so that $J_{-}=c(x) / K(x)$ where $c(x)$ is the concentration of the solution in equilibrium with the layer characterized by a given undersaturation degree $x$. The saturated surface coverage, $\Gamma_{0}$, corresponds to a layer of infinitely long chains, $N \rightarrow \infty$. The chemical potential balance between the adsorbed layer and the bulk, equation (2.19), gives

$$
\rho(x) \equiv c(x) / c^{*} \sim N^{A^{\prime}} \exp (-N / G) \sim N^{0.25} \exp (-N / G)
$$

where $G \sim x^{\frac{1}{1-2 \nu}}$. The flux of desorbing monomers is then

$$
J_{-} \sim G^{-2+\gamma_{2 \mathrm{D}}-\gamma_{1}-2 \nu} N^{1+\gamma_{1}-\gamma_{2 \mathrm{D}}} \exp (-N / G) \sim N^{049} G^{-2.66} \exp (-N / G)
$$

When the layer is not in equilibrium with the bulk solution, we assume that the desorption flux does not depend on the actual bulk solution concentration but that it depends only on the degree of undersaturation. We thus make the assumption that the desorption flux is still given by equation (4.4). Let us also recall that at each time we assume that the adsorbed layer has an equilibrium structure and thus that the inward and outward fluxes are small enough to allow reequilibration.

4.1. Formation OF AN AdSORBed LAYER. - We consider a moderately starved layer, $g^{*}<G \ll N$, exposed to a dilute polymer solution. In this situation, the desorption is exponentially small and can be neglected. The adsorption rate equation can then be written as

$$
\frac{\mathrm{d} x}{\mathrm{~d} t}=-J_{+}=-\rho N^{024} x^{15.1}
$$

where we have used for simplicity the numerical estimates of the exponents. The undersaturation degree decreases with time as

$$
x \sim N^{-0017}(\rho t)^{-0071}
$$


Thus the surface coverage approaches the saturation limit according to a very slow power law: a decrease of $x$ by a factor of 2 implies an increase of the relaxation time by a factor of $\sim 20000$. Taking into account the direct relation between the surface coverage and the cut-off tail length, $G$ we can rewrite this equation as

$$
t \sim \frac{1}{\rho} N^{-024} G^{2.48}
$$

Note that in the region of interest, $G>N^{0.90}$, the characteristic relaxation time $t=t(G)$ is always longer than both the relaxation time of a terminal $G$-part which is spread over the adsorbing wall, $\tau_{\mathrm{R}} \sim G^{2}$, and the characteristic time corresponding to motions of largest tails and loops, $\tau_{Z} \sim G^{3 \nu} \simeq G^{1.76}$.

Substituting $G \sim N$ in equation (4.7) we get the longest relaxation time for the formation of an adsorbed layer: $t_{\text {ads }} \sim \frac{1}{\rho} N^{3 / 2+3 \nu-\frac{\gamma}{2 \nu}+\gamma-\gamma_{2 D}} \sim \frac{1}{\rho} N^{224}$. In the regime $t \gg t_{\text {ads }}$ the surface coverage approaches equilibrium according to a simple exponential law: $\Gamma(\infty)-\Gamma(t) \propto$ $\exp \left(-t / t_{\text {ads }}\right)$.

Note that the adsorption time $t_{\mathrm{ads}}$ thus obtained is always longer than the longest time of conformational relaxation, $\tau_{\mathbf{R}} \sim N^{2}$ provided that the solution is dilute $(\rho \lesssim 1)$. This justifies the local equilibrium assumption.

4.2. DeSORPTION. - We now consider the reverse situation of an adsorbed layer first prepared in equilibrium with a dilute solution of concentration $c_{0}$, and then suddenly exposed to a pure solvent. The surface coverage decreases due to an outward flux, $J_{-}$. The desorption rate equation is written as:

$$
\frac{\mathrm{d} x}{\mathrm{~d} t}=J_{-} \sim N^{049} G^{-266} \exp (-N / G)
$$

The initial condition $x(0)=x_{0}$ is determined by the equilibrium with a solution at a concentration $c_{0}$. We define $y_{0}=N / G_{0}$ or equivalently $x_{0} \sim\left(y_{0} / N\right)^{2 \nu-1}$. The equilibrium condition gives

$$
y_{0} \simeq 0.25 \ln N-\ln \rho_{0}
$$

where $\rho_{0} \equiv c_{0} / c^{*}$. The variable $y_{0}$ increases thus only logarithmically when the molecular weight is increased or when the concentration is decreased. Note that although $x_{0}$ is small, it does not vanish: the surface coverage of a saturated layer of finite chains, $\Gamma_{e}=\Gamma_{0}\left(1-x_{0}\right)$, is slightly smaller than the limiting value $\Gamma_{0}$ corresponding to an infinite molecular weight.

The undersaturation degree increases as

$$
x \simeq N^{1-2 \nu}\left[y_{0}+\ln \left(1+t / t_{\mathrm{des}}\right)\right]^{2 \nu-1}
$$

where

$$
t_{\mathrm{des}} \sim \frac{y_{0}^{-4+\gamma_{2 \mathrm{D}}-\gamma_{1}}}{\rho_{0}} N^{3 / 2+3 \nu+\gamma-\gamma_{2 \mathrm{D}}-\frac{\gamma}{2 \nu}} \sim \frac{y_{0}^{-3.48}}{\rho_{0}} N^{2.24}
$$

This gives the following asymptotic limits :

$$
x \simeq x_{0}\left[1+(2 \nu-1) y_{0}^{-1} \ln \left(1+t / t_{\mathrm{des}}\right)\right]
$$

for $t / t_{\text {des }} \ll \exp \left(y_{0}\right) \sim N^{025} / \rho_{0}$, and

$$
x \sim N^{1-2 \nu}\left[\ln \left(1+t / t_{\mathrm{des}}\right)\right]^{2 \nu-1}
$$

for $t / t_{\text {des }} \gg \exp \left(y_{0}\right)$. 
Note that in the region $t \gg t_{\text {des }}$ the rate of desorption is primarily governed by the effective desorption barrier, $N \Delta \mu=N / G$, which is large for a starved layer. The desorption time of a fraction $x$ of initially adsorbed chains is exponentially large (for $x \gg x_{0}$ ):

$$
t \sim t_{\mathrm{des}} \exp \left(a N x^{\frac{1}{2 \nu-1}}\right)
$$

where $a$ is a numerical factor.

The desorption is very slow on a large time-scale. The characteristic fraction of the chains that effectively desorb is of order $N^{1-2 \nu} \simeq N^{-0176}$ (apart from a logarithmic factor which cannot be large: for example $t \sim 10^{10} t_{\text {des }}$ corresponds to a logarithmic factor $\left[\ln \left(1+t / t_{\text {des }}\right]^{2 \nu-1}\right.$ in the r.h.s. of Eq. (4.13) equal to $\simeq 1.7$ ). Therefore the fraction of the desorbed chains is always small unless the time of desorption is extremely large.

4.3. Exchange Solution-Adsorbate. - The third basic experiment is the exchange experiment where a layer prepared by a preliminary adsorption of radioactively labelled polymers is then exposed to a solution of chemically identical non-labelled chains. We call $\Gamma^{*}$ the surface excess of the labelled chains, and $\Gamma_{\mathrm{e}}$ the total surface excess. The total coverage is constant since the adsorbed layer remains in equilibrium with the bulk solution during the whole exchange process: the total fluxes $J_{+}$and $J_{-}$are equal and constant. Using equations (4.2), (2.19) and (4.9) we get

$$
J_{\mathrm{e}}=J_{+}=J_{-} \sim \rho_{0} N^{-242} y_{0}^{266}
$$

The labelled species are being gradually washed out from the layer. The corresponding outward rate is proportional to $J_{\mathrm{e}}$ and to the fraction of labelled chain, $\Gamma^{*} / \Gamma_{\mathrm{e}}$, in the layer:

$$
\frac{\mathrm{d} \Gamma^{*}}{\mathrm{~d} t}=-\frac{\Gamma^{*}}{\Gamma_{\mathrm{e}}} J_{\mathrm{e}}
$$

The solution of the last equation is

$$
\Gamma^{*}(t)=\Gamma^{*}(0) \exp \left(-t / \tau^{*}\right)
$$

where

$$
\tau^{*} \sim \frac{1}{\rho_{0}} y_{0}^{-266} N^{242}
$$

The exchange time is inversely proportional to the bulk concentration as was first predicted by De Gennes [10]. Note also that generally the exchange (washing out of the labelled polymers) is much faster than the desorption, which is exponentially slow (see Eq. (4.14)). On the other hand, the exchange time, $\tau^{*}$, is longer than the maximum time corresponding to adsorption, $t_{\text {ads }}$ :

$$
\tau^{*} / t_{\mathrm{ads}} \sim y_{0}^{082} N^{0} 18
$$

As pointed out by De Gennes [10], the difference between $\tau^{*}$ and $t_{\mathrm{des}}$ is similar to the difference between cooperative and self-diffusion rates: the driving force for an exchange is of order of $k T$, whereas a formation of an adsorbed layer is generally associated with a much stronger force $\sim N \Delta \mu=N / G$.

\section{Concluding Remarks}

We have discussed the kinetics of adsorption of a dilute polymer solution onto a solid wall. We have described the penetration of a new chain in the layer as a two-step process. The entry 
process is controlled by the excluded volume interaction of the incoming chain with the already adsorbed chains. The spreading process requires the expulsion of already adsorbed chains to maintain nearly constant the total number of monomers in direct contact with the surface during the penetration of a new chain. A detailed analysis of the Rouse-Zimm dynamics of an entering chain coupled with the effective barrier due to excluded volume interactions provides a possibility to predict the molecular weight dependences of all the relevant characteristic times for adsorption, desorption and exchange processes. In particular, we showed that desorption is normally much slower than exchange, which in turn is slower than adsorption, in qualitative agreement with earlier predictions of De Gennes. However, the particular molecular weight dependences of the relaxation times are new and different from those obtained by De Gennes. There are three reasons for this discrepancy: 1) we explicitly take into account the structure of tails and loops in the adsorbed layer that leads to an effective repulsion of the chain end points from the adsorbing surface; 2) we assumed Rouse-Zimm, rather than reptation, dynamics of polymer chains; 3 ) we consider the rate of penetration of new chains in terms of purely dynamical quantity, the effective resistance of the potential barrier created by already adsorbed polymers, in contrast to the quasi-equilibrium consideration of De Gennes.

Thus it is not surprising that we also get a few qualitatively new results: we showed that it is the spreading stage that controls the adsorption rate if the layer is nearly saturated (the cut-off tail size $\left.G>N^{0.84}\right)$. In the case of stronger starvation of the layer $\left(G<N^{0.84}\right)$, we predict no barrier for adsorption at all (i.e., the adsorption rate is controlled by diffusion of incoming chains only in this regime). We also predict that end-entry is the dominant mechanism of chain penetration for a saturated or moderately starved layer $\left(G>N^{089}\right)$, whereas hairpin entry is dominant for more starved layers.

In particular, in the very late stages the adsorption is limited by the spreading process and the chain penetration is always done by end entry. The resulting potential barrier experienced by a penetrating chain is higher than that previously calculated by De Gennes $[10,11,27]$. This leads to a much slower kinetics of formation of the adsorbed layer the surface excess reaches the saturation value only as a very slow power law of time $t^{-0} 071$ (see Eq. (4.6)). The longest relaxation time for the formation of an adsorbed layer is inversely proportional to the monomer bulk concentration.

Chain desorption induced by washing out an adsorbed layer by a pure solvent is predicted to be an extremely slow process. The typical fraction of chains that can actually be desorbed is in principle rather small, $f \sim N^{1-2 \nu} \simeq N^{-0176}$. In practice, this does not however always give small numbers: even for large $N \sim 10^{4}$ we get $f \sim 20 \%$. The desorption time for an appreciably larger amount of polymer grows exponentially with the molecular weight (Eq. (4.14)), and probably cannot be achieved in experiments. This conclusion is in agreement with the experimental fact that an adsorbed layer can not be taken off by washing with pure solvent $[2,17]$.

We have also considered the exchange of polymer chains between an adsorbed layer of labelled chains and an unlabelled solution. The final picture agrees with that previously suggested by De Gennes [10]. The characteristic time of exchange is inversely proportional to the solution concentration in agreement with De Gennes's theory [10] and the experimental observations [28, 29].

Other new predictions correspond to the molecular weight dependencies of the characteristic times. In particular we show that the exchange time scales as $\tau^{*} \sim N^{2.42}$, whereas the terminal time for the formation of a (saturated) adsorbed layer is $t_{\text {ads }} \propto N^{224}$. For large $N$ the exchange time is thus much longer than the adsorption time [10].

The main limitation of the present theory is that it is based on the assumption that the polymer chains are not entangled. Definitely, the effect of topological constraints must be 
important for adsorbed layers formed by very high molecular weight polymers. A future work is thus required to generalize this approach and to discuss the kinetics of formation of entangled adsorbed layers.

\section{Acknowledgments}

This work was performed during the stay of one of the authors (A.N.S.) at Universite Louis Pasteur in Strasbourg (Institut Charles Sadron). A.N.S. would like to thank the University and the Ministry of Higher Education of France for the opportunity to stay in Strasbourg.

\section{References}

[1] De Gennes P.G., Macromolec 14 (1981) 1637.

[2] De Gennes P.G., Adv Collond Interface Scr. 27 (1987) 189.

[3] Fleer G. J., Cohen Stuart M A., Scheutjens J.M.H.M., Cosgrove T and Vincent B., Polymers at Interfaces (Chapman and Hall, London, 1993).

[4] De Gennes P.G., C.R. Acad. Scr. (Parıs) II 294 (1982) 1317.

[5] Auvray L. and Cotton J.P., Macromolec. 20 (1987) 202.

[6] Klein J., Proceedings of the "Liquids at Interfaces" Conference, Les Houches, France, J. Charvolin, J.-F. Joanny and J. Zinn-Justin Eds. (North Holland, Amsterdam, 1990).

[7] Semenov A. and Joanny J.-F., "Structure of Adsorbed Polymer Layers: Loops and Tails", Europhys. Lett. 29 (1995) 279.

[8] Frantz P. and Granick S., Macromolec. 27 (1994) 2553.

[9] Rossi G. and Pincus P.A., Macromolec. 22 (1989) 276.

[10] De Gennes P.G., "Molecular Conformations and Dynamics of Macromolecules in Condensed Systems", Studies in Polymer Science, Vol. 2, M. Nagasawa Ed. (Elsevier Sci. Publishers B.V., Amsterdam, 1988) p. 315.

[11] De Gennes P.G., Proceedings of an International Symposium on New Trends in Physics and Physical Chemistry of Polymers (Plenum Press, N.Y., 1989) p. 9.

[12] De Gennes P G., Scaling Concepts in Polymer Physics (Cornell Univ. Press, Ithaca, NY, 1978).

[13] Des Cloizeaux J, and Jannınk G., Les Polymères en solution: Leurs propriétés et leur structure (Les Editions de Physique, Les Ulis, France, 1987).

[14] Duplantier B., J. Stat. Phys. 54 (1989) 581.

[15] De Gennes P.G , C R. Acad. Scı. (Parıs) II 301 (1985) 1399.

[16] Zinn-Justin J., Quantum Field Theory and Critical Phenomena (Clarendon Press, Oxford, 1989).

[17] Cohen Stuart M., Cosgrove T. and Vincent B , Adv. Collozd Interface Scı. 24 (1986) 143.

[18] Semenov A.N., in preparation.

[19] De Gennes P.G., J. Chem Phys. 55 (1971) 572.

[20] Doi M. and Edwards S.F., The Theory of Polymer Dynamics (Clarendon Press, Oxford, 1986).

[21] The effect of excluded volume interactions on the reptation dynamics was analyzed in reference [22]. The results, when applied to a two-dimensional case $(d=2)$, immediately lead to equation (3.15) for the disentanglement time.

[22] Semenov A.N., Physica A 171 (1991) 517.

[23] Duplantier B., J. Phys. A 19 (1986) L1009.

[24] Van Kampen N., Stochastic Processes in Physics and Chemistry (North Holland, Amsterdam, 1992). 
[25] Scheutjens J. and Fleer G.J., J. Phys. Chem. 84 (1980) 178.

[26] Cohen Stuart M.A , Waajen F.H W.H., Cosgrove T., Vincent B. and Crowley T.L., Macromolec 17 (1984) 1825.

[27] De Gennes P.G., C.R. Acad. Scr. (Parzs) II 301 (1985) 1399.

[28] Pefferkorn E., Carroy A. and Varoqu R,, J. Polym Scr. (Phys.) 23 (1985) 1997.

[29] Varoqui R. and Pefferkorn E., Progr. Collord. Polym Scı. 83 (1990) 96. 